\title{
A flood map based DOI decoding method for block detector: A GATE simulation study
}

\author{
Han Shi ${ }^{\mathrm{a}}$, Dong $\mathrm{Du}^{\mathrm{a}}{ }^{*}$, Zhihong $\mathrm{Su}^{\mathrm{b}}$ and Qiyu Peng ${ }^{\mathrm{c}}$ \\ ${ }^{a}$ Tsinghua University, Welding Building Room 111, Beijing, China \\ ${ }^{b}$ Southern Medical University, Guangzhou, China \\ ${ }^{c}$ Lawrence Berkeley National Laboratory, CA, USA
}

\begin{abstract}
Positron Emission Tomography (PET) systems using detectors with Depth of Interaction (DOI) capabilities could achieve higher spatial resolution and better image quality than those without DOI. Up till now, most DOI methods developed are not cost-efficient for a whole body PET system. In this paper, we present a DOI decoding method based on flood map for low-cost conventional block detector with four-PMT readout. Using this method, the DOI information can be directly extracted from the DOI-related crystal spot deformation in the flood map. GATE simulations are then carried out to validate the method, confirming a DOI sorting accuracy of $85.27 \%$. Therefore, we conclude that this method has the potential to be applied in conventional detectors to achieve a reasonable DOI measurement without dramatically increasing their complexity and cost of an entire PET system.
\end{abstract}

Keywords: Positron emission tomography, depth of interaction, GATE simulation

\section{Introduction}

Positron Emission Tomography (PET), a widely applied imaging method in metabolism, neuroscience, and molecular imaging [1-3], generally uses detector modules consisting of discrete crystal array and photo detectors [4]. For crystals decoding, the flood map of the discrete crystal array is used to generate a LookUp Table. As traditional method only decodes the location of the discrete crystal, in some situations, DOI information existing in the flood map would be omitted when traditional method is employed. Traditional DOI method is adding complicated extra design for DOI in the detector, which would result in increased design complexity and reduced energy and time performance $[1,5,6]$.

It has been proved that DOI information does exist in the flood map. In Yang's dual ended detector study, the flood map obtained by one end PSAPD experiences obvious changes while decoding spots get elongated in shape when the interaction depth increases [7]. Besides, some crystal mapping spots in the flood map have similar "ghosting" phenomenon as that in Wei's PMT Quadrant Sharing detector decoding study [8]. Up till now, for single crystal and single detector photoelectric sensor design, or single ended readout detector, no reports have been found to directly estimate gamma ray reaction depth using flood map.

${ }^{*}$ Corresponding author: Dong Du, Department of Mechanical Engineer, Welding Building Room 113, Tsinghua University, Beijing, China. Tel./Fax: +86 01062783862; E-mail: dudong@tsinghua.edu.cn. 
In nuclear medical imaging research, the Monte Carlo simulation software GATE (Geant4 Application for Tomographic Emission) is extensively used for simulations [9]. Because optical models of Geant 4 have been added, GATE can now perform simulations for visible photons transmission, which helps to accurately predict the performance of PET detectors [10], especially in specular reflection models [11]. As a result, GATE acts as a suitable tool for DOI research.

In this paper, we propose a DOI identification method based on single-ended readout mapping decoding position, and GATE simulations are carried out to confirm the validity of this method.

\section{Method}

\subsection{Detector module and decoding position analysis}

As shown in Figure 1(a), traditional detector module is composed of a crystal array and 4 PMTs (Photo-Multiplier Tube). A light guide with several slots filled by reflective materials is coupled with the crystal array and the PMTs on two ends, respectively. In order to decode the interaction crystal, Anger Logic is used to generate the crystal decoding map called flood map, shown as Figure 1(b). In the flood map, each spot is corresponding to a discrete crystal element in the array. As for one spot, its decoding position of interaction in the same crystal varies due to interaction depth uncertainty, photon transmission randomness and signal acquisition error. As a result, for each crystal in the flood map, the decoding map gives a spot rather than a point.

Figure 2 analyzes the effects of different DOIs on decoding position in detail. Figure 2(a) gives one dimensional representation of the detector. As long as interaction occurs in the crystal (no matter at a, $\mathrm{b}$ or c), large amount of low-energy visible photons will be generated. Before being detected by the PMTs, these photons need to pass through the crystal and the light guide. During this process, the photons will get bounced back and forth by the ESR reflectors, resulting in different photon distributions on 4 PMT detection surfaces. Based on the distribution, one-dimensional decoding position can be calculated using Eq. (1). Figure 2(b) shows the simulated distribution when interactions occur at a and c depth.

$$
\begin{aligned}
& \text { position }=\mathrm{PMT}_{\mathrm{A}} /\left(\mathrm{PMT}_{\mathrm{A}}+\mathrm{PMT}_{\mathrm{B}}\right) \\
& \mathrm{PMT}_{\mathrm{A}}=\int_{0}^{25} f(x) \mathrm{d} x
\end{aligned}
$$
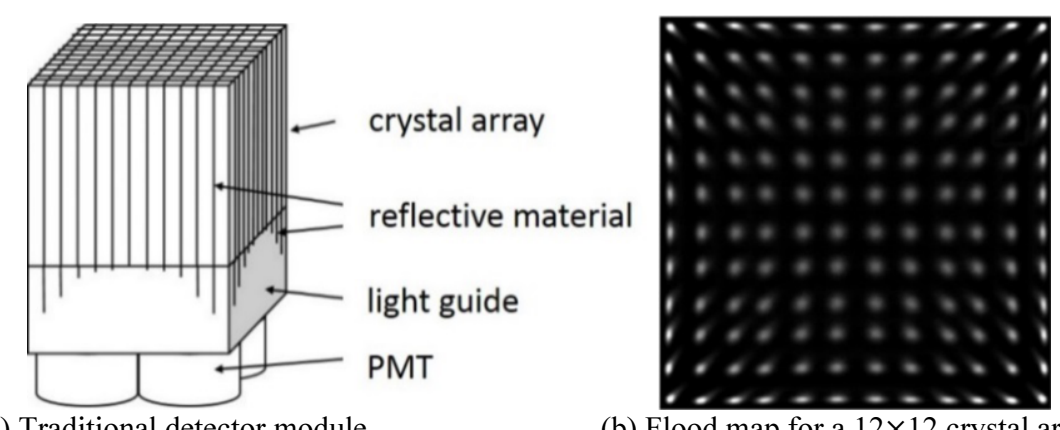

(a) Traditional detector module.

(b) Flood map for a $12 \times 12$ crystal array

Fig. 1. Traditional detector module and the decoding map. 

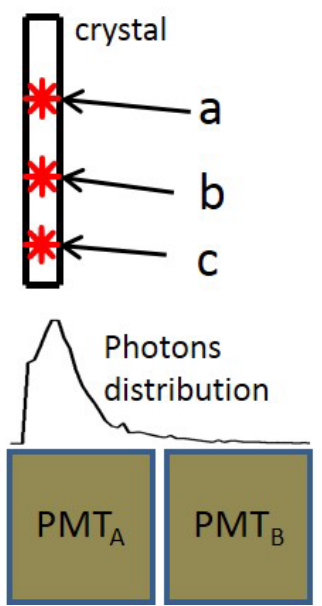

(a) Photons detection of light sharing based detector module in one dimension.

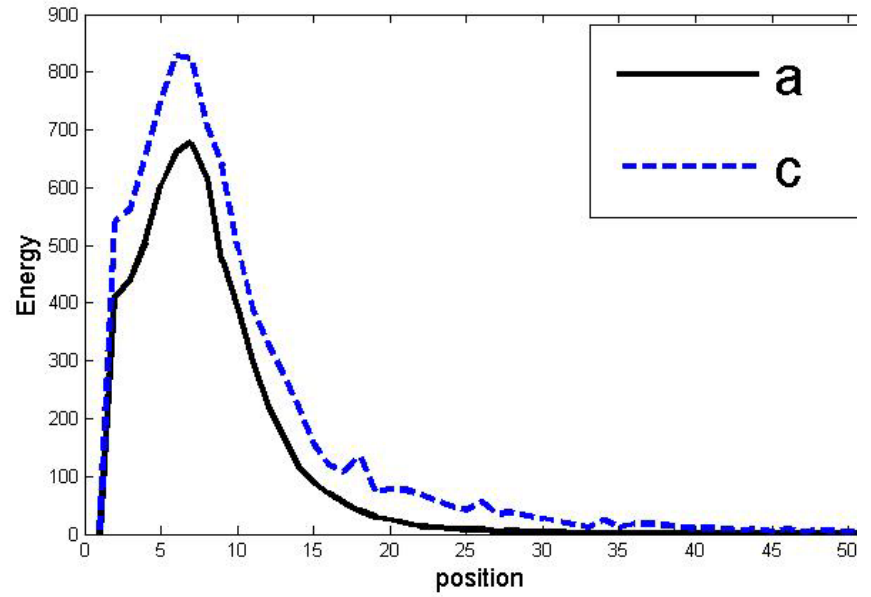

(b) Photon distributions of a and $\mathrm{b}$ interaction depths.

Fig. 2. Depth of interaction affects decoding position.

$$
\operatorname{PMT}_{\mathrm{B}}=\int_{25}^{50} f(x) \mathrm{d} x
$$

where $f(x)$ is the intensity distribution function of the photons. $\mathrm{PMT}_{\mathrm{A}}$ and $\mathrm{PMT}_{\mathrm{B}}$ are the energy intensity obtained by the two PMTs. Obviously, we have position $\mathrm{a}_{\mathrm{a}}<\operatorname{position}_{\mathrm{c}}$, and the decoding position of $\mathrm{b}$ is between a and c. Therefore, the reason why the DOI information is included in the decoding position is theoretically explained.

\subsection{DOI-encoding method}

To summarize the DOI measurement method based on mapping decoding position, a flow chart is given in Figure 3. In order to establish the relation between DOI and decoding position, a large number of interactions $(10,000$ counts in the simulation) are simulated at different depths using Anger Logic method to determine the decoding position/coordinates. For each depth, a probability density function is obtained to describe the decoding position distribution. As a result, a series of twodimensional probability density functions $f_{l}(x, y)$ are generated. Afterwards, in the flood map, for each pixel $(x, y)$ of the spot corresponding to the crystal, the expectation of interaction depth, $\mathrm{d}$, can be calculated using

$$
d=\frac{\sum\left[l \times f_{l}(x, y) \times \exp (-\mu l)\right]}{\sum\left[f_{l}(x, y) \times \exp (-\mu l)\right]}
$$

where, $\exp (-u l)$ is used for attenuation correction. $l$ and $u$ are the interaction depth and the crystal linear attenuation coefficient respectively. For example, the linear attenuation coefficient of LYSO is 87 $\mathrm{cm}^{-1}[12]$. 


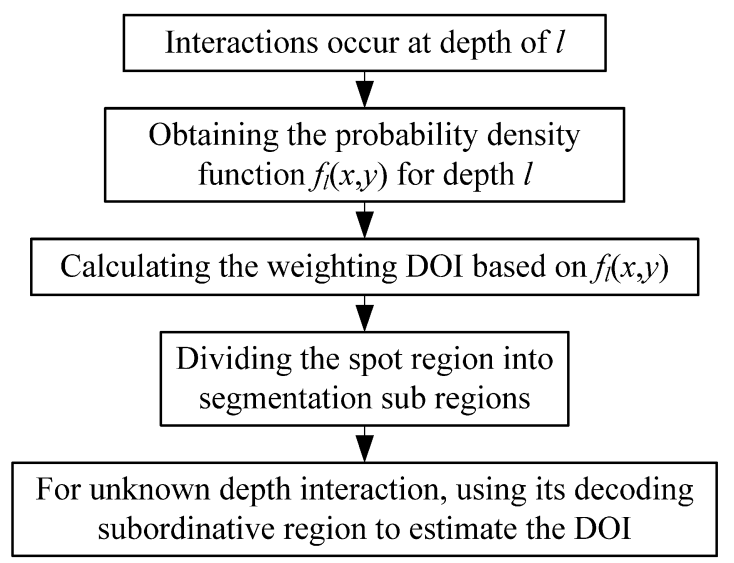

Fig. 3. Flow chart of DOI identification method based on mapping decoding position.

Table 1

GATE simulation parameters

\begin{tabular}{ll}
\hline Simulation parameter & Value \\
\hline Scintillation material & LYSO \\
Crystal size & $4 \mathrm{~mm} \times 4 \mathrm{~mm} \times 25 \mathrm{~mm}$ \\
Light yield & 26,000 photons per MeV \\
Linear attenuation coefficient & $0.87 \mathrm{~cm}-1 @ 511 \mathrm{keV}$ \\
Visible light attenuation length & $42 \mathrm{~cm}$ \\
Reflector material & ESR film VM2000 \\
Reflector thickness & $0.15 \mathrm{~mm}$ \\
Reflective coefficient & 0.985 \\
Light guide attenuation length & $42 \mathrm{~cm}$ \\
PMT type & Hamamatsu R9800 \\
PMT photon detection efficiency & $25 \%$ \\
\hline
\end{tabular}

Therefore, the conversion map from decoding position to DOI can be obtained. Lastly, according to the DOI measurement requirements, the spot area is divided into several sub areas. As for unknown depth interactions, their DOIs can be estimated using their decoding position.

\subsection{Simulation method}

In this paper, we employ GATE for carrying out simulations with basic simulation parameters listed in Table 1. Besides, the reflection type is set to $100 \%$ "specular lobe distribution" and the surface type is set to "dielectric metal" to simulate the ESR film. The surface finish is set to "polished" with a deviation of reflective angle of $0.31^{\circ}$ as measured by Janecek with a Dektak 150 surface profilometer on a mechanically polished surface [8]. $4 \mathrm{~mm} \times 4 \mathrm{~mm} \times 25 \mathrm{~mm}$ LYSO crystal is selected as the scintillator, whose light yield is 26,000 photons per MeV. Absorbing length $\left(\mu_{a b}=50 \mathrm{~cm}\right)$ and Rayleigh scattering length $\left(\mu_{R s}=260 \mathrm{~cm}\right)$ were constrained to a total attenuation length $\left(\mu_{a t}=42 \mathrm{~cm}\right)$ for LYSO [13]. The attenuation length in light guide is assumed the same. Linear attenuation coefficient of LYSO is 87 $\mathrm{cm}-1$. The reflector is ESR film with the reflective coefficient of 0.985 [11]. The ratio of effective photons to light yield is 0.25 , which included the light collection efficiency and photodetector quantum efficiency. The effective photons is calculated as $26000 \times \frac{511}{1000} \times 25 \%=3321 \mathrm{In}$ order to short the 
computing time, the number of effective photons is directly used in simulation, and 3321 photons are used to represent the light output of Gamma ray with energy of $511 \mathrm{KeV}$. A Linux shell program is developed to call the GATE macro programs and Octave programs to generate and analyze the GATE simulation data, respectively.

\subsection{Sorting accuracy}

The Sorting Accuracy is used to assess the DOI method. For example, for a two-bin DOI method, the crystal is divided into two layers: the upper layer and the lower layer. For the depth belonging to the upper layer, a large number of events are simulated. The events sorted as the upper layer by the DOI method are true classification, and the events sorted as the lower layer are false classification. The sorting accuracy of the depth is the result of dividing the true classification by the total events. Total sorting accuracy of the DOI method is the average of the sorting accuracy of different depths.

\section{Results and analysis}

Figure 4 shows the conversion map from decoding position to DOI as calculated by weighting method.

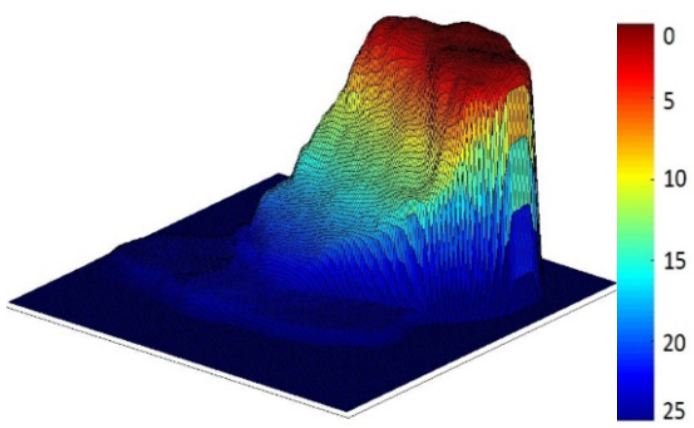

Fig. 4. Conversion map between decoding position and DOI.

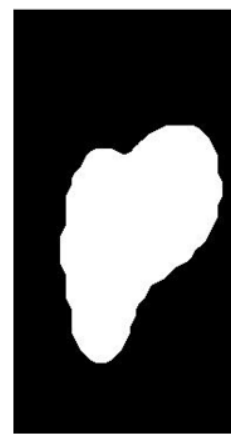

(a)

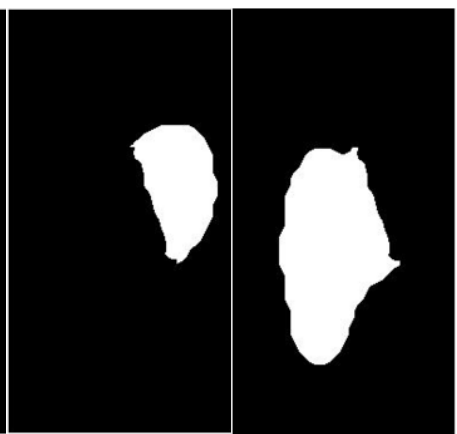

(b) (c)

Fig. 5. Two layers of DOI segmentation for the crystal. (a) Spot region (conversion region). (b) Sub region corresponding to the interactions occur in the upper layer. (c) Sub region corresponding to interactions occur in the lower layer. 


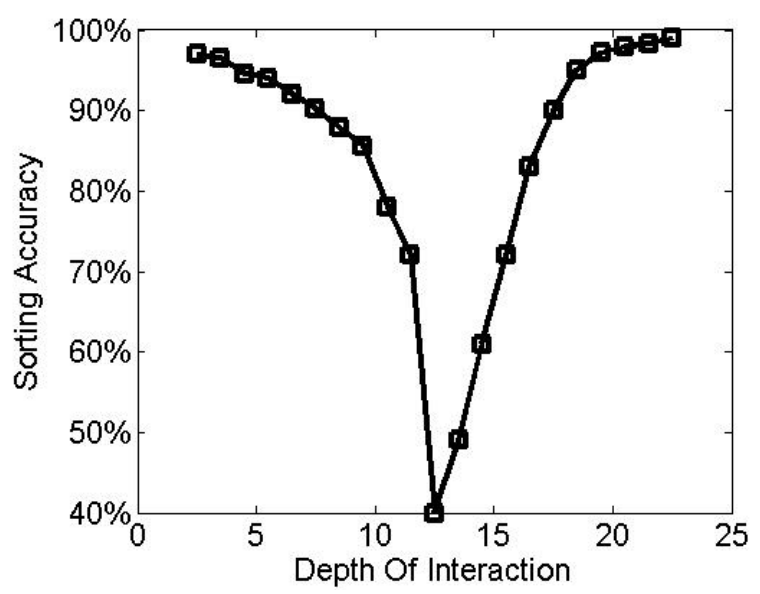

Fig. 6. Sorting accuracy as a function of depth.

For the most basic DOI measurement, namely dividing the crystal into two layers, a threshold equal to half of the crystal length is used to segment the conversion map and divide the spot region (Figure 5(a)) into two sub regions, as shown in Figures 5(b) and 5(c).

The segmentation region in Figure 5 is used to validate the accuracy of proposed DOI decoding method. In GATE simulations, the interaction depth varies from 2.5 to $22.5 \mathrm{~mm}$, with a $1 \mathrm{~mm}$ gradient. Figure 6 summarizes the DOI sorting accuracy. As we can see, the sorting accuracy is high when the interactions occur at the two ends of the crystal, while it is low when interactions occur in the middle. Specifically, when the depth is $12.5 \mathrm{~mm}$, due to the attenuation correction effect, DOI sorting accuracy is lower than $50 \%$. However, attenuation correction improves the sorting accuracy when interactions occur in the upper half crystal, considering that the interaction taking to a exponential decay distribution along the crystal. According to the exponential distribution, weighted average of the 21 depths sorting accuracy is $85.27 \%$.

\section{Conclusion}

In this paper, we propose a DOI decoding method based on flood map for low-cost conventional block detectors with four-PMT readout. Conversion map between decoding position and DOI needs to be established first, followed by dividing the spot area mapping one crystal in flood map into different sub regions, which correspond to different DOIs. As validated by the sorting accuracy of $85.27 \%$ in GATE based simulations, we conclude that this method can successfully realize the DOI measurement. In the future, we will assemble detectors and conduct relevant experiments to get some experimental evidence and try to apply the method into PET detector.

\section{Acknowledgement}

This work was supported in part by China Scholarship Council (201206210102) and International Science \& Technology Cooperation Program of China (2013DFB30270). 


\section{References}

[1] E. Yoshida, H. Yamashita, H. Tashima, S. Kinouchi, H. Murayama, M. Suga and T. Yamaya, Design study of the DOIPET scanners with the x'tal cubes toward sub-millimeter spatial resolution, Journal of Medical Imaging and Health Informatics 3 (2013), 131-134.

[2] H.P. Ren, H. Yang, B.R. Ma, S.Z. Chen and W.K. Wu, A novel magnetic resonance-positive emission image registration based on morphology, Bio-Medical Materials and Engineering 13 (2003), 187-196.

[3] J. Jiang, X. Lin, J. Wen, Z. Huang and Z. Yan, A method of semi-quantifying beta-AP in brain PET-CT 11C-PiB images, Bio-Medical Materials and Engineering 23 (2003), S1419-1425.

[4] J.G. Rogers, A.J. Taylor, M.F. Rahimi, R. Nutt, M. Andreaco and C.W. Williams, An improved multicrystal 2-D bgo detector for pet, IEEE Transactions on Nuclear Science 39 (1992), 1063-1068.

[5] H. Peng and C.S. Levin, Recent developments in PET instrumentation, Current Pharmaceutical Biotechnology 11 (2010), 555-571.

[6] S.T. Liu, S.H. An, H.D. Li, C. Wang, R.A. Ramirez, Y.X. Zhang, H. Baghaei and W.H. Wong, A dual-layer TOF-DOI detector block for whole-body PET, IEEE Transactions on Nuclear Science 59 (2012), 1805-1808.

[7] Y.F. Yang, Y.B. Wu, J.Y. Qi, S.S. James, D. Huini, P.A. Dokhale, K.S. Shah, R. Farrell and S.R. Cherry, A prototype PET scanner with DOI-encoding detectors, Journal of Nuclear Medicine 49 (2008), 1132-1140.

[8] Q.Y. Wei, S. Wang, T.Y. Ma, F. Ma, T.T. Dai, T.P. Xu, Z.X. Wu, Y.J. Jin and Y.Q. Liu, Influence factors of two dimensional position map on photomultiplier detector block designed by quadrant sharing technique, Nuclear Science and Techniques 22 (2011), 224-229.

[9] G. Santin, D. Strul, D. Lazaro, L. Simon, M. Krieguer, M.V. Martins, V. Breton and C. Morel, GATE: A Geant4-based simulation platform for PET and SPECT integrating movement and time management, IEEE Transactions on Nuclear Science 50 (2003), 1516-1521.

[10] D.J. van der Laan, D.R. Schaart, M.C. Maas, F.J. Beekman, P. Bruyndonckx and C.W.E. van Eijk, Optical simulation of monolithic scintillator detectors using GATE/GEANT4, Physics in Medicine and Biology 55 (2010), 1659-1675.

[11] M. Janecek and W.W. Moses, Simulating scintillator light collection using measured optical reflectance, IEEE Transactions on Nuclear Science 57 (2010), 964-970.

[12] H. Peng, P.D. Olcott, V. Spanoudaki and C.S. Levin, Investigation of a clinical PET detector module design that employs large-area avalanche photodetectors, Physics in Medicine and Biology 56 (2011), 3603-3627.

[13] I. Vilardi, A. Braem, E. Chesi, F. Ciocia, N. Colonna, F. Corsi et al., Optimization of the effective light attenuation length of YAP: Ce and LYSO: Ce crystals for a novel geometrical PET concept, Nuclear Instruments \& Methods in Physics Research Section a-Accelerators Spectrometers Detectors and Associated Equipment 564 (2006), 506-514. 